\title{
Metallothionein crypt-restricted immunopositivity indices (MTCRII) correlate with aberrant crypt foci (ACF) in mouse colon
}

\author{
ET Donnelly', H Bardwell', GA Thomas 2,6, ED Williams², M Hoper', P Crowe', WG McCluggage ${ }^{3}$, \\ M Stevenson ${ }^{4}$, DH Phillips ${ }^{5}$, A Hewer $^{5}$, MR Osborne ${ }^{5}$ and FC Campbell ${ }^{*, 1}$ \\ 'Departments of Surgery, Centre for Cancer Research and Cell Biology, Queen's University of Belfast, Clinical Sciences Building, Grosvenor Road, Belfast \\ BTI 2 6BJ, Northern Ireland, UK; ${ }^{2}$ Strangeways Research Laboratories, Worts Causeway, Cambridge CBI 8RN, UK; ${ }^{3}$ Department of Pathology, Centre for \\ Cancer Research and Cell Biology, Queen's University of Belfast, Belfast BTI 2 6BJ, Northern Ireland, UK; ${ }^{4}$ Department of Epidemiology, Centre for Cancer \\ Research and Cell Biology, Queen's University of Belfast, Belfast BT I 2 6BJ, Northern Ireland, UK; ${ }^{5}$ Section of Molecular Carcinogenesis, Institute of Cancer \\ Research, Brookes Lawley Building, Cotswold Road, Sutton, Surrey SM2 5NG, UK
}

Metallothionein (MT) crypt-restricted immunopositivity indices (MTCRII) are colonic crypt stem cell mutation markers that may be induced early and in abundance after mutagen treatment. Metallothionein is the endogenous reporter gene for MTCRII, but is not typically implicated in the classical pathway of colorectal tumorigenesis. Hence, the oncological relevance of MTCRII is unclear. This study tests the hypothesis that MTCRII induced by $N$-methyl-N-nitrosourea (MNU) and lambda carrageenan ( $\lambda$ CgN) associate with aberrant crypt foci (ACF) in mouse colon. Undegraded $\lambda \mathrm{CgN}$ and $\mathrm{MNU}$ were tested alone and in combination against MTCRII and $\mathrm{ACF}$ in Balb/c mice, at 20 weeks after the start of treatment. MTCRII were unaffected by $\lambda \mathrm{CgN}$ alone. Combined $\lambda \mathrm{CgN} / \mathrm{MNU}$ treatments induced greater MTCRII $(P<0.0 \mathrm{I})$ as well as greater number $(P<0.00 \mathrm{I})$ and crypt multiplicity $(P<0.0 \mathrm{I})$ of $A C F$ than MNU alone. MTCRII were approximately I0-fold more numerous than ACF, although linear correlations were observed between these parameters $(r=0.732 ; P<0.0 I)$. MTCRII are induced by $\lambda \mathrm{CgN} / \mathrm{MNU}$ interactions in sufficient numbers to provide statistical power from relatively small sample sizes and correlate with ACF formation. MTCRIl could thus provide the basis for a novel mediumterm murine bioassay relevant to early-stage colorectal tumorigenesis.

British Journal of Cancer (2005) 92, 2160-2165. doi:I0.1038/sj.bjc.6602633 www.bjcancer.com

Published online 3I May 2005

(c) 2005 Cancer Research UK

Keywords: stem cell; mutation; mixture

Humans are exposed to mixtures of genotoxic and nongenotoxic environmental chemicals that may be linked to cancer (Burkart and Jung, 1998; Minamoto et al, 1999). Robust biomarkers of somatic stem cell mutation and mutant clonal expansion may provide cancer surrogates that are useful for risk assessment. Acquired mutation of a selectable endogenous reporter gene like glucose-6-phosphate dehydrogenase (G6PD) within a colonic crypt stem cell induces a crypt-restricted phenotype change (Griffiths et al, 1988; Park et al, 1995; Kuraguchi et al, 1997). Stable, cryptrestricted immunopositivity for metallothionein (MT) is a more recently described stem cell mutation marker for mouse colon that can be assayed in paraffin-fixed tissue sections and has been validated against the G6PD assay (Cook et al, 2000). MT-immunopositive crypt frequency has shown a dose response to three different chemical mutagens (Jasani et al, 1998; Cook et al, 2000; Donnelly et al, 2004), which strengthens the evidence that it is a somatic mutation marker.

We have previously shown that a model genotoxic/nongenotoxic chemical mixture, comprising $N$-methyl- $N$-nitrosourea (MNU) and

*Correspondence: Professor FC Campbell;

E-mail: F.C.Campbell@qub.ac.uk

${ }^{6}$ Thomas' current address: South West Wales Cancer Institute, Singleton Hospital, Sketty, Swansea SA2 8QA, UK

Revised 21 April 2005; accepted 22 April 2005; published online 3 I May 2005 undegraded lambda carrageenan $(\lambda \mathrm{CgN})$, have interactive effects upon MT crypt-restricted immunopositivity indices (MTCRII), including frequency and size of MT-immunopositive foci and total number of MT-immunopositive crypts (Donnelly et al, 2004). However, the role of MT mutation in the classical molecular pathway of colorectal cancer is uncertain and thus the oncological relevance of MTCRII is unclear.

This study assesses MTCRII induced by similar $\mathrm{MNU} / \lambda \mathrm{CgN}$ regimens as in our previous investigation (Donnelly et al, 2004) against aberrant crypt foci (ACF) in mouse colon. MTCRII develop within 4-6 weeks of mutagen exposure (Jasani et al, 1998; Cook et al, 2000). ACF represent the earliest morphological stage of colorectal tumorigenesis (McLellan et al, 1991). Some early ACF may regress (Shpitz et al, 1996), although large ACF that develop at late intervals after mutagen treatment may have greater risk of dysplasia and cancer (Papanikolaou et al, 2000). In this study therefore, assays were conducted at 20 weeks after mutagen treatment to enable assessment of MTCRII against large or persistent ACF.

\section{MATERIALS AND METHODS}

Chemicals, reagents, animals and treatment regimens were as described previously (Donnelly et al, 2004). Anti-MT primary antibody (mouse anti-horse monoclonal E9, isotype:IgG1) and peroxidase-conjugated rabbit anti-mouse immunoglobulins 
were obtained from DAKO Ltd, Ely, Cambridgeshire, UK (Dako M0639 and P0161 respectively), as described previously (Jasani et al, 1998; Cook et al, 2000). Methylene blue was obtained from BDH Chemicals Ltd, Poole, Dorset, UK (BDH 34048).

\section{Animals and treatment regimes}

Female adult Balb/c mice, aged 6-8 weeks, were obtained from Harlan UK Ltd, Bicester, Oxon, UK, divided into groups of five or 10, ear-punched and placed in coded stainless-steel wire cages, maintained and fed as outlined previously (Donnelly et al, 2004). Individual animal weights and group fluid and AIN-76 diet consumption were assessed daily, during weekdays.

\section{Treatment groups}

In all, 90 female adult Balb/c mice aged 6-8 weeks were divided into 11 groups of five or 10 that received no MNU, MNU (62.5 $\mathrm{mg} \mathrm{kg}^{-1}$ dissolved in dimethylsulphoxide (DMSO)) alone or in combination with 1 or $4 \% \lambda \mathrm{CgN}$ as follows:

Group $1(n=5)$ : Drinking water only for 20 weeks (water only control).

Group $2(n=5)$ : Single intraperitoneal (i.p.) injection of vehicle (DMSO), then drinking water for 20 weeks (vehicle control).

Group $3(n=5)$ : Continuous $1 \% \lambda \mathrm{CgN}$ only for 20 weeks.

Group $4(n=5)$ : Continuous $4 \% \lambda \mathrm{CgN}$ only for 20 weeks.

Group $5(n=10)$ : MNU $62.5 \mathrm{mg} \mathrm{kg}^{-1}$ i.p., then drinking water only for 20 weeks.

Group $6(n=10)$ : MNU $62.5 \mathrm{mg} \mathrm{kg}^{-1}$ i.p. and $1 \% \lambda \mathrm{CgN}$ for 7 days during week 1 , then drinking water until 20 weeks.

Group $7(n=10)$ : MNU $62.5 \mathrm{mg} \mathrm{kg}^{-1}$ i.p. and $4 \% \lambda \mathrm{CgN}$ for 7 days during week 1 , then drinking water until 20 weeks.

Group $8(n=10)$ : MNU $62.5 \mathrm{mg} \mathrm{kg}^{-1}$ i.p. and three 7 -day treatments of $1 \% \lambda \mathrm{CgN}$ during weeks 1,4 and 7 . Drinking water was given between and after $\lambda \mathrm{CgN}$ treatments until 20 weeks.

Group $9(n=10)$ : MNU $62.5 \mathrm{mg} \mathrm{kg}^{-1}$ i.p. and three 7 -day treatments of $4 \% \lambda \mathrm{CgN}$ during weeks 1,4 and 7 . Drinking water was given between and after $\lambda \mathrm{CgN}$ treatments until 20 weeks.

Group $10(n=10)$ : MNU $62.5 \mathrm{mg} \mathrm{kg}^{-1}$ i.p. and continuous $1 \%$ $\lambda \mathrm{CgN}$ treatment until 20 weeks.

Group $11(n=10)$ : MNU $62.5 \mathrm{mg} \mathrm{kg}^{-1}$ i.p. and continuous $4 \%$ $\lambda \mathrm{CgN}$ treatment until 20 weeks.

In combined regimens, MNU was administered after the first 5 days of $\lambda \mathrm{CgN}$ treatment.

\section{Welfare considerations and weight index}

Animal welfare considerations were strictly in accordance with OECD guidelines (OECD, 2002). Animals were weighed daily and weight index was calculated as the ratio at study completion relative to weight at study start. Values for mean weight index were compared between treatment groups, at study completion.

\section{Assay of ACF}

All assays of ACF were blinded to treatment and carried out after colonic retrieval at 20 weeks after the initiation of treatment. Colons were carefully pinned flat on a cork mat, painted with $0.1 \%$ methylene blue and left at room temperature for $10 \mathrm{~min}$. Assay of
ACF was performed using a dissecting microscope at $\times 40$ magnification and the following parameters were recorded:

(i) ACF number: Assessed as the total number of ACF per colon or per $10^{4}$ colonic crypts.

(ii) ACF size (crypt multiplicity): Crypt multiplicity was determined as the number of aberrant crypts per ACF.

Colons were then 'Swiss-rolled' on the cork mat, with the ileocaecal junction at the centre of the roll, fixed in neutral formal saline for $48 \mathrm{~h}$, and embedded in paraffin wax blocks.

\section{Assay of MTCRII}

All assays of MTCRII were blinded to treatment and carried out 20 weeks after the initiation of treatment. MTCRII were assayed as described previously (Donnelly et al, 2004). Briefly, paraffinembedded sections ( $4 \mu \mathrm{m}$ thickness) were cut at 10 levels (L1L10), $100 \mu \mathrm{m}$ apart through the 'Swiss-rolled' colon. One section from each level was stained using a standard indirect immunoperoxidase technique for MT, while endogenous peroxidase activity was blocked using $3 \%$ hydrogen peroxide in methanol. Slides were incubated with an anti-MT primary antibody (E9, isotype: IgG1; $100 \mu \mathrm{l}$ per slide) (DAKO Ltd, Ely, Cambridgeshire, UK). The secondary antibody used was horseradish peroxidase-conjugated rabbit anti-mouse immunoglobulin (Dako catalogue no. P0161). Negative control sections were incubated either in the absence of antibody, in normal mouse serum $(1: 1000)$, or with an irrelevant antibody of the same IgG subclass (1:1000) (IgG1; Dako catalogue product code X0931). These were consistently negative. Positive control sections included mouse colon previously treated by $N$-ethyl- $N$-nitrosourea (ENU; $250 \mathrm{mg} \mathrm{kg}^{-1}$ ), which induces MTimmunopositive crypts (Cook et al, 2000). Sections were washed and $100 \mu \mathrm{l}$ of the chromogen 3,3'-diaminobenzidine tetrahydrochloride was added before counter-staining with Harris haematoxylin. The frequency and size of MT-immunopositive foci as well as total number of MT-immunopositive crypts were assessed as follows:

(i) MT-immunopositive single crypts or patches of $\geqslant 2$ contiguous MT-immunopositive crypts were recognised by their dark brown stain against a haematoxylin background and were assessed in transverse or longitudinal section through the crypt lumen. Each single or contiguous patch of $\geqslant 2$ MTimmunopositive crypts was considered to represent a single mutant focus.

(ii) The size of each MT-immunopositive patch was assessed by the number of contiguous MT-immunopositive crypts within the patch. Patches were recorded as doubles, triples or greater ( $n=2,3$, etc., MT-immunopositive crypts).

(iii) The total number of MT-immunopositive crypts per mouse colon was determined by the sum of single immunopositives and all immunopositives within patches.

Endpoints of the (i) frequency of MT-immunopositive foci (ii) number of MT-immunopositive patches and (iii) the total number of MT-immunopositive crypts were expressed as the number per $10^{4}$ total crypts, in mouse colon.

\section{Data analysis}

Serial weight data were available in individual mice. The weight index was calculated as the weight at study completion relative to weight at study start, expressed as a percentage. Between-group differences of weight index were assessed by one-way ANOVA. Descriptive statistics applied to weight index were expressed as mean \pm standard deviation (mean \pm s.d.). Group data were available for consumption of food and fluid, which were assessed in grams or ml per kg body weight, respectively. Descriptive statistics 
were expressed as mean \pm s.d. To achieve a normal distribution, MTCRII and ACF data from each treatment group were log transformed to ensure a normal distribution and assessed by a probability plot of residuals. Transformed data were analysed by univariate ANOVA. Duncan post hoc tests were applied to assess differences between specific treatment regimens. Differences of MT-immunopositive patch formation between $\mathrm{MNU}$ alone and all combinations of $\lambda \mathrm{CgN} / \mathrm{MNU}$ were assessed by Student's $t$-test. Correlations between MTCRII and ACF data were investigated by Pearson's product moment coefficient. SPSS for Windows (version 11) was used for statistical analysis (SPSS Inc., Chicago, Il, USA).

\section{RESULTS}

\section{Food, fluid intake and body weight}

In all, 11 treatment groups of mice ( $n=90$ total) received wateror vehicle-only controls, high- or low-dose $\lambda \mathrm{CgN}$ alone or in combination with $\mathrm{MNU}\left(62.5 \mathrm{mg} \mathrm{kg}^{-1}\right.$ i.p.). $\lambda \mathrm{CgN}$ was given in single or recurrent short- or long-term patterns of exposure. Group values for fluid, food intake and weight index are shown in Table 1. No significant between-group differences of weight index were observed at study completion (Table 1).

Table I Effects of 20-week treatments on group fluid and food consumption and weight index

\begin{tabular}{|c|c|c|c|c|}
\hline Treatment regimes & Mice (n) & $\begin{array}{c}\text { Group } \\
\text { fluid } \\
\text { intake } \\
\text { ( } \mathrm{ml} \mathrm{g}^{-1} \\
\text { body } \\
\text { weight) }\end{array}$ & $\begin{array}{c}\text { Group } \\
\text { food } \\
\text { intake } \\
\text { ( } \mathrm{gg}^{-1} \\
\text { body } \\
\text { weight) }\end{array}$ & $\begin{array}{l}\text { Weight } \\
\text { index }\end{array}$ \\
\hline I. Control (water) only & 5 & 0.165 & 0.184 & $13272+$ \\
\hline 2. DMSO vehicle only & 5 & 0.163 & 0.192 & $|33.7| \pm 2$ \\
\hline 3. Continuous $1 \% \lambda \mathrm{CgN}$ & 5 & 0.172 & 0.174 & $|26.5| \pm 3$ \\
\hline 4. Continuous $4 \% \lambda \mathrm{CgN}$ & 5 & 0.113 & 0.184 & $132.09 \pm 4.7$ \\
\hline 5. MNU $\left(62.5 \mathrm{mg} \mathrm{kg}^{-1}\right)$ & 10 & 0.152 & 0.161 & $126.58 \pm 6$ \\
\hline 6. $\mathrm{MNU}+1 \times 7$ day $1 \% \lambda \mathrm{CgN}$ & 10 & 0.151 & 0.170 & $130.49 \pm 6$ \\
\hline 7. $\mathrm{MNU}+\mathrm{I} \times 7$ day $4 \% \lambda \mathrm{CgN}$ & 10 & 0.149 & 0.165 & $126.43 \pm 5$ \\
\hline 8. $\mathrm{MNU}+3 \times 7$ day $1 \% \lambda \mathrm{CgN}$ & 10 & 0.149 & 0.167 & $133.29 \pm 6.9$ \\
\hline 9. $\mathrm{MNU}+3 \times 7$ day $4 \% \lambda \mathrm{CgN}$ & 10 & 0.122 & 0.165 & $129.95 \pm 3$ \\
\hline 10. MNU+continuous $1 \% \lambda \mathrm{CgN}$ & 10 & 0.137 & 0.164 & $130.22 \pm 4$ \\
\hline II. MNU+continuous $4 \% \lambda \mathrm{CgN}$ & 10 & 0.114 & 0.165 & $125.41 \pm 6$ \\
\hline
\end{tabular}

$\mathrm{DMSO}=$ dimethylsulphoxide; $\lambda \mathrm{CgN}=$ lambda carrageenan; $\mathrm{MNU}=\mathrm{N}$-methyl- $\mathrm{N}$ nitrosourea.

\section{Treatment effects upon MTCRII}

Group values for MTCRII, including total number of MTimmunopositive crypts, MT-immunopositive patch formation and frequency of MT-immunopositive foci, are shown in Table 2. The total number of MT-immunopositive crypts was increased by $>25$-fold in excess of that of vehicle alone, by MNU $\left(62.5 \mathrm{mg} \mathrm{kg}^{-1}\right)$ treatment, but was unaffected by $\lambda \mathrm{CgN}$ treatment alone. Data analysis by one-way between-group ANOVA with the Duncan post hoc test allowed division of results into statistically different subsets. Combined $\lambda \mathrm{CgN} / \mathrm{MNU}$ regimens induced significantly greater total number of MT-immunopositive crypts compared to MNU alone or treatments lacking $\mathrm{MNU}(P<0.01$; Table 2). Significant incremental differences were observed between treatment subsets $(\mathrm{A}-\mathrm{C})$, where $\mathrm{A}$ represents treatment groups $1-4, \mathrm{~B}$ represents groups 5 and 6 and $C$ represents groups 8 and 11 . Treatment groups 7,9 and 10 overlapped subsets $B$ and $C$ (Figure 1A).

Significant between-group differences in the frequency of patches of $\geqslant 2$ contiguous MT-immunopositive crypts were also observed $(P<0.05$; ANOVA $)$. Significant incremental differences were observed between three treatment subsets $(A-C)$, where A represents treatment groups $1-4, \mathrm{~B}$ represents group 5 and $\mathrm{C}$ represents groups 7 and 9. Treatment groups 6, 8, 10 and 11 overlapped subsets B and C (Figure 1B). Over 95\% of mutant patches involved only two contiguous mutant crypts. The frequency of large MT-immunopositive patches $(\geqslant 3$ contiguous immunopositive crypts) was $0.38 \pm 0.5$ per $10^{4}$ total crypts for MNU alone (group 5) vs $1.12 \pm 1.01$ per $10^{4}$ total crypts for all MNU/ $\lambda \mathrm{CgN}$ treatment groups $(P=0.002)$. All patches of $\geqslant 4 \mathrm{MT}$ immunopositive crypts were observed in combined $\lambda \mathrm{CgN} / \mathrm{MNU}$ treatment groups.

\section{Effect of treatment regimes on ACF frequency}

The administration of MNU led to a significant increase in ACF numbers by about 10 -fold in excess of that of DMSO vehicle alone. ACF data were expressed either as a number per $10^{4}$ total colonic crypts (Figure $2 \mathrm{~A}$ ) or per mouse colon (Table 3 ). $\lambda \mathrm{CgN}$ treatment alone led to a small significant increase in ACF size, in terms of crypt multiplicity but had no significant effect on ACF number. Combined $\lambda \mathrm{CgN} / \mathrm{MNU}$ regimens significantly increased $\mathrm{ACF}$ number and size $(P<0.001$; ANOVA). Post hoc analysis demonstrated significant incremental differences in ACF number between five homogeneous treatment subsets $(\mathrm{A}-\mathrm{E})$, where A represents treatment groups 1-4, B represents treatment group 5, C represents groups 6 and 7, D represents group 8 and $\mathrm{E}$ represents groups 9 and 11. Group 10 overlapped subsets C and D (Figure 2A; Table 3). Significant effects of treatment on ACF size were also observed $(P<0.01$; Figure $2 \mathrm{~B})$ with incremental differences in

Table 2 Treatment effects upon MTCRII

\begin{tabular}{|c|c|c|c|c|c|}
\hline Treatment & Mice (n) & Total crypt no. & $\begin{array}{c}\text { Total MT-immunopositive } \\
\text { crypt number }\end{array}$ & $\begin{array}{c}\text { Patches } \geqslant 2 \text { MT- } \\
\text { immunopositive crypts }\end{array}$ & $\begin{array}{l}\text { Frequency of MT- } \\
\text { immunopositive foci }\end{array}$ \\
\hline I. Water only & 5 & $36433 \pm 2053$ & $1.134 \pm 0.34$ & $0.10 \pm 0.06$ & $1.130 \pm 0.33$ \\
\hline 2. DMSO only & 5 & $33292 \pm 2888$ & $0.974 \pm 0.29$ & $\overline{0}$ & $0.97 \pm 0.29$ \\
\hline 3. Continuous $1 \% \lambda \mathrm{CgN}$ & 5 & $37025 \pm 2091$ & $0.88 \pm 0.32$ & 0 & $0.88 \pm 0.33$ \\
\hline 4. Continuous $4 \% \lambda \mathrm{CgN}$ & 5 & $38657 \pm 1149$ & $1.31 \pm 0.32$ & 0 & $1.31 \pm 0.32$ \\
\hline 5. MNU only $\left(62.5 \mathrm{mg} \mathrm{kg}^{-1}\right)$ & 10 & $29750 \pm 1925$ & $37.66 \pm 2.93$ & $5.54 \pm 0.91$ & $31.73 \pm 2.66$ \\
\hline 6. $\mathrm{MNU}+\mathrm{I} \times 7$-day cycle $1 \% \lambda \mathrm{CgN}$ & 10 & $27715 \pm 1715$ & $46.54 \pm 5.63$ & $6.76 \pm 1.10$ & $38.60 \pm 5.18$ \\
\hline 7. $\mathrm{MNU}+\mathrm{I} \times 7$-day cycle $4 \% \lambda \mathrm{CgN}$ & 10 & $32539 \pm 1543$ & $52.31 \pm 4.82$ & $9.61 \pm 0.78$ & $39.39 \pm 4.72$ \\
\hline 8. $\mathrm{MNU}+3 \times 7$-day cycles $1 \% \lambda \mathrm{CgN}$ & 10 & $30651 \pm 209 \mid$ & $59.85 \pm 6.91$ & $8.27 \pm 1.56$ & $51.46 \pm 6.81$ \\
\hline 9. $\mathrm{MNU}+3 \times 7$-day cycles $4 \% \lambda \mathrm{CgN}$ & 10 & $30640 \pm 3176$ & $52.47 \pm 7.80$ & $9.62 \pm 0.67$ & $43.79 \pm 5.89$ \\
\hline 10. MNU+continuous $1 \% \lambda \mathrm{CgN}$ & 10 & $31372 \pm 1832$ & $47.86 \pm 5.96$ & $8.00 \pm 1.14$ & $38.14 \pm 5.11$ \\
\hline I I. MNU+continuous $4 \% \lambda \mathrm{CgN}$ & 10 & $23450 \pm 1007$ & $56.23 \pm 5.41$ & $5.82 \pm 0.69$ & $49.98 \pm 5.09$ \\
\hline
\end{tabular}

MTCRII = metallothionein crypt-restricted immunopositivity indices; $M T=$ metallothionein; DMSO = dimethylsulphoxide; $\lambda \mathrm{CgN}=$ lambda carrageenan; $\mathrm{MNU}=\mathrm{N}-\mathrm{methyl}-\mathrm{N}$ nitrosourea. 

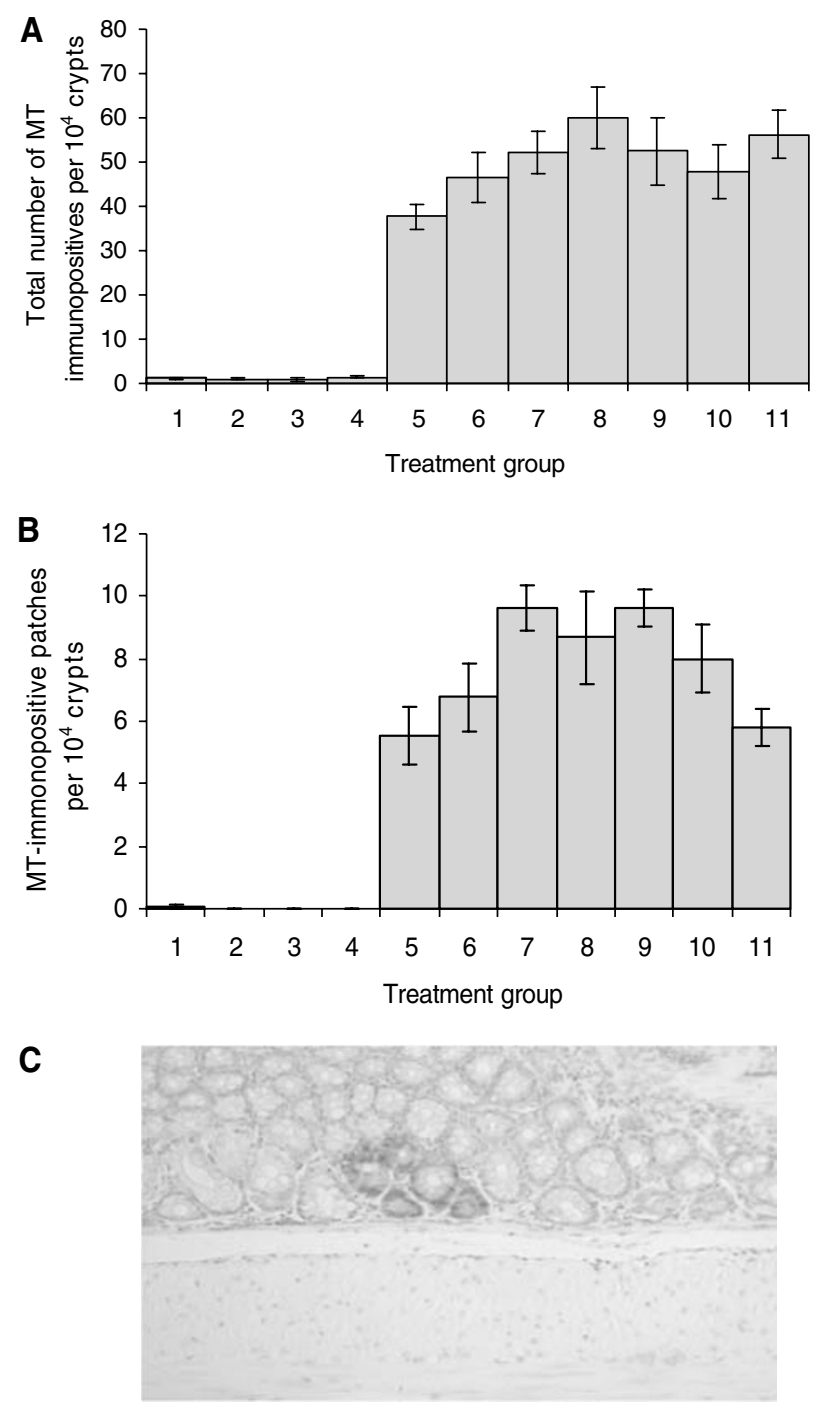

Figure I Treatment effects upon MTCRII. (A) Total MT-immunopositive crypt number per $10^{4}$ crypts in each treatment group. Significant between-group treatment differences were observed $(P<0.01)$. The Duncan post hoc test identified three distinct homogeneous treatment subsets $(A-C)$, showing significant incremental differences in total mutation load: (A) Groups I-4, distilled water, DMSO or $\lambda \mathrm{CgN}$ only (I and 4\%); (B) Groups 5 and 6, MNU only or MNU and one 7 day cycle of I\% $\lambda \mathrm{CgN}$; (C) Groups 8 and II, MNU and three 7 day cycles of $1 \% \lambda \mathrm{CgN}$ or MNU and continuous 4\% $\lambda \mathrm{CgN}$. Groups 7, 9 and 10 overlapped subsets B and C. (B) Formation of patches of $\geqslant 2$ contiguous MT immunopositive crypts in each treatment group. The Duncan posthoc test identified three distinct homogeneous treatment subsets $(A-C)$, showing significant incremental differences in mutant (MT immunopositive) patch formation: (A) Groups I -4, Distilled water, DMSO or $\lambda \mathrm{CgN}$ only (I and 4\%); (B) Group 5, MNU alone; (C) Groups 7 and 9, MNU and one or three 7 day cycles of $4 \%$ $\lambda \mathrm{CgN}$. Groups 6, 8, 10 and II overlapped subsets B and C. (C) Large MT immunopositive patch. An example of a large patch comprising 5 MT immunopositive crypts, from a combined $\lambda \mathrm{CgN} / \mathrm{MNU}$ treatment group.

crypt multiplicity within ACF between five treatment subsets (A-E), where A represents treatment groups 1 and 2, B represents 3 and 4, C represents group 5 and 6, D groups 9 and 11 and $\mathrm{E}$ represents group 8 . Groups 7 and 10 overlapped subsets D and E.

\section{Correlations between MTCRII and ACF}

Assessments of MTCRII and ACF were conducted in all treatment groups $(1-11)$. Linear correlations were observed between total
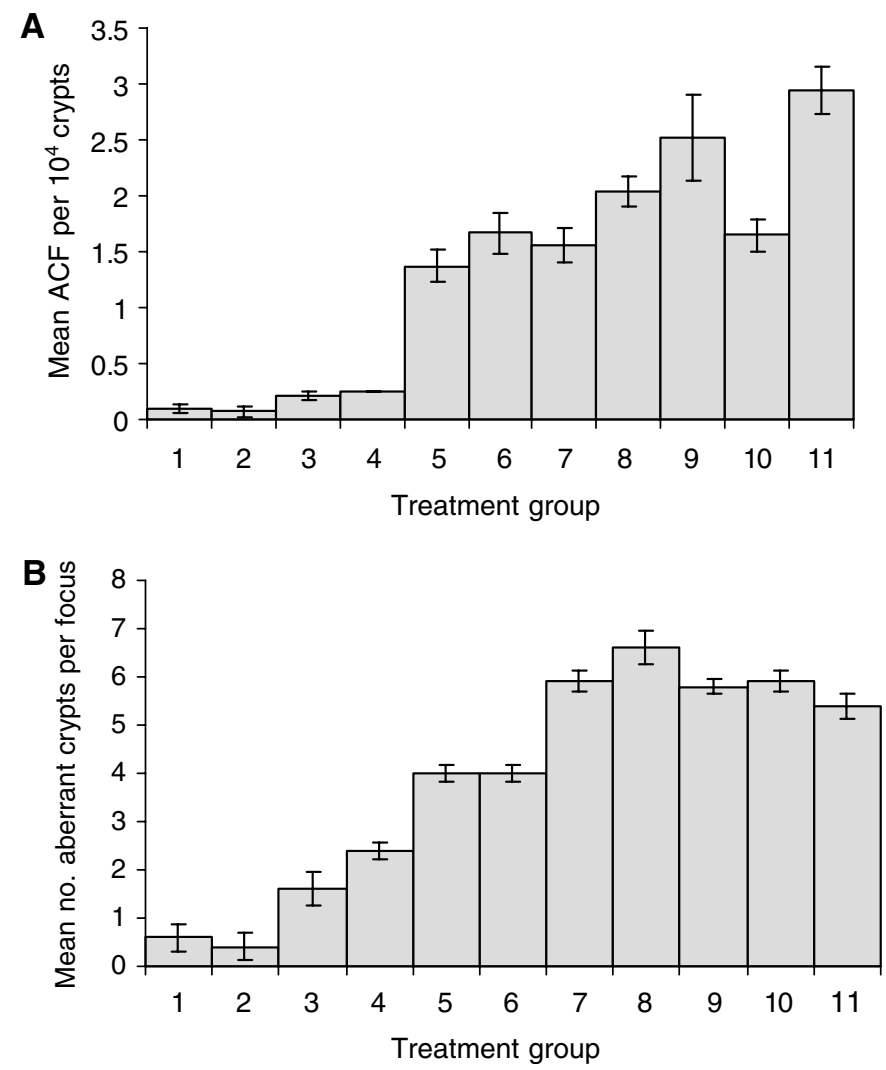

Figure 2 Treatment effects on ACF. (A) Effects of treatment regimens on ACF number in murine colon. One-way analysis of variance demonstrated significant between-group differences in numbers of ACF per $10^{4}$ colonic crypts $(P<0.00 \mathrm{I})$. The Duncan post hoc test identified 5 homogeneous treatment subsets $(A-E)$, showing significant incremental differences in frequency of ACF formation: (A) Groups 1-4, Distilled water, DMSO or $\lambda \mathrm{CgN}$ only (I and 4\%); (B) Group 5, MNU alone; (C) Groups 6 and 7, MNU and one 7 day cycle of I or $4 \% \lambda \mathrm{CgN}$; (D) Group 8, $\mathrm{MNU}$ and three 7-day cycles of $1 \% \lambda \mathrm{CgN}$; (E) Groups 9 and II, MNU and either three 7 -day cycles of $4 \% \lambda \mathrm{CgN}$ or continuous $4 \% \lambda \mathrm{CgN}$. These subsets showed significant incremental differences of mean ACF per $10^{4}$ colonic crypts. Group 10 (MNU and $3 \times 7$-day cycles of $4 \% \lambda \mathrm{CgN}$ ) overlapped subsets $C$ and D. (B) Effects of treatment regimens on size of ACF (crypt multiplicity). One-way analysis of variance demonstrated significant between-group differences in numbers of aberrant crypts per focus \{crypt multiplicity\} $(P<0.0 \mathrm{I})$. The Duncan post hoc test identified 5 homogeneous treatment subsets $(A-E)$, showing significant incremental differences in crypt multiplicity: (A) groups I and 2, distilled water alone or DMSO alone; (B) groups 3 and 4 , continuous treatment with I or $4 \%$ $\lambda \mathrm{CgN}$ alone; (C) groups 5 and $6, \mathrm{MNU}$ alone or $\mathrm{MNU}$ and $\mathrm{I} \times 7$-day cycle of $1 \% \lambda \mathrm{CgN}$; (D) groups 9 and I I, MNU and either $3 \times 7$-day cycles of $4 \%$ $\lambda \mathrm{CgN}$ or continuous $4 \% \lambda \mathrm{CgN}$; (E) group $8, \mathrm{MNU}$ and three 7 -day cycles of $1 \% \lambda \mathrm{CgN}$. Groups 7 and 10 (MNU and I $\times 7$-day cycle of $4 \% \lambda \mathrm{CgN}$ or continuous $1 \% \lambda \mathrm{CgN}$ ) overlapped subsets $\mathrm{D}$ and $\mathrm{E}$.

MT-immunopositive crypt number and ACF number per $10^{4}$ crypts $(r=0.732 ; P<0.01)$ (Figure $3 \mathrm{~A}$ ) and ACF size, in terms of the number of aberrant crypts per focus $(r=0.84 ; P<0.01)$ (Figure 3B).

\section{DISCUSSION}

Colonic tumorigenesis involves acquisition of mutations or heritable epigenetic events, affecting growth control or differentiation genes within crypt stem cells, progression to premalignant stages including ACF (Bird and Good, 2000) and ultimate invasive 
Table 3 Treatment effects upon number and size of ACF

\begin{tabular}{|c|c|c|c|c|c|}
\hline Group & Treatment & Mice (n) & No. of ACF per colon & No. of ACF per $10^{4}$ crypts & No. of aberrant crypts per $A C F$ \\
\hline 2 & DMSO only & 5 & $0.20 \pm 0.20$ & $0.07 \pm 0.04$ & $0.40 \pm 0.25$ \\
\hline 5 & MNU only & 10 & $3.90 \pm 0.23$ & $1.37 \pm 0.015$ & $4.00 \pm 0.17$ \\
\hline 6 & $\mathrm{MNU}+\mid \times 7$-day cycle $1 \% \lambda \mathrm{CgN}$ & 10 & $5.00 \pm 0.29$ & $1.67 \pm 0.18$ & $4.00 \pm 0.16$ \\
\hline 7 & $\mathrm{MNU}+\mathrm{I} \times 7$-day cycle $4 \% \lambda \mathrm{CgN}$ & 10 & $4.90 \pm 0.23$ & $1.56 \pm 0.14$ & $5.90 \pm 0.21$ \\
\hline 10 & $\mathrm{MNU}+$ continuous $1 \% \lambda \mathrm{CgN}^{\circ}$ & 10 & $5.00 \pm 0.25$ & $1.65 \pm 0.14$ & $5.90 \pm 0.22$ \\
\hline । & $\mathrm{MNU}+$ continuous $4 \% \lambda \mathrm{CgN}$ & 10 & $6.80 \pm 0.29$ & $2.95 \pm 0.21$ & $5.38 \pm 0.26$ \\
\hline
\end{tabular}

$\mathrm{ACF}=$ aberrant crypt foci; $\mathrm{DMSO}=$ dimethylsulphoxide; $\lambda \mathrm{CgN}=$ lambda carrageenan; $\mathrm{MNU}=\mathrm{N}$-methyl-N-nitrosourea.

A

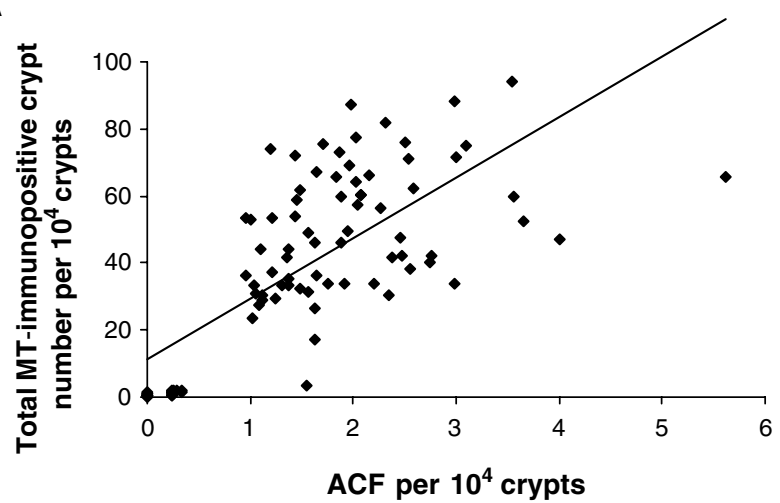

B

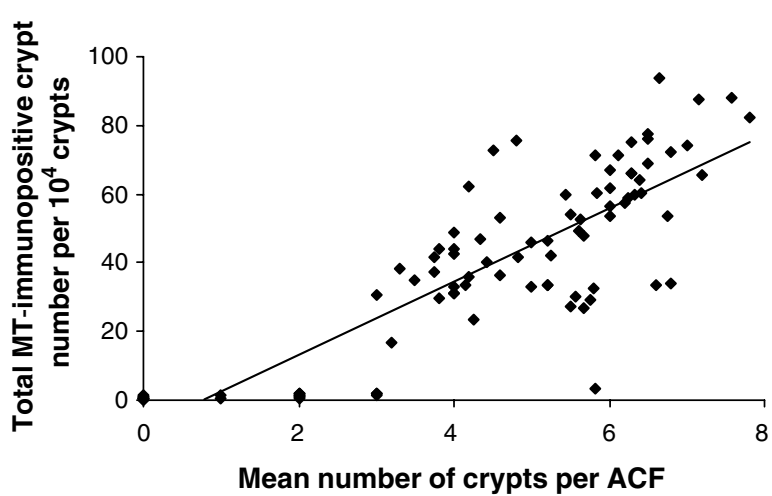

Figure 3 Correlations between MTCRII and ACF. (A) Total MTimmunopositive crypt number vs number of ACF per $10^{4}$ crypts. Correlation between total MT-immunopositive crypt number and ACF per $10^{4}$ crypts in all treatment groups $(r=0.732 ; P<0.0$ I by Pearson's product moment test). (B) Total MT-immunopositive crypt number vs size of ACF. Correlation between total MT-immunopositive crypt number per $10^{4}$ crypts and ACF size in all treatment groups $(r=0.84 ; P<0.01$ by Pearson's product moment test).

carcinoma. Since these events are stochastic, a higher stem cell mutation rate may accelerate distinct stages of this process (Herrero-Jimenez et al, 1998). Robust biomarkers of stem cell mutation may thus provide useful surrogates of tumorigenesis. Metallothionein crypt-restricted immunopositivity indices provide a stem cell mutation marker that is initiated by mutagen exposures, yet mimics sporadic tumorigenesis because it occurs in widely scattered single crypts or foci throughout the otherwise normal colon (Cook et al, 2000). Since the relationship of MT crypt-restricted immunopositivity to tumorigenesis was unclear, we assessed the relationship between MTCRII and ACF frequency, in mice treated by $\lambda \mathrm{CgN}$ and MNU.

The present study uses a similar combinatorial design, involving a single MNU treatment $\left(62.5 \mathrm{mg} \mathrm{kg}^{-1}\right)$ together with single, repeated or continuous exposures to low- $(1 \%)$ or high- $(4 \%)$ dose $\lambda \mathrm{CgN}$, to that of our previous study (Donnelly et al, 2004). In the present study however, follow-up and continuous $\lambda \mathrm{CgN}$ treatment were continued for longer term (20 weeks). The present study supports our earlier work and shows that $\lambda \mathrm{CgN}$ alone does not significantly affect MTCRII, but enhances MNU effects upon this end point (Donnelly et al, 2004). However, sequential or prolonged $\lambda \mathrm{CgN}$ exposure to 20 weeks was associated with the development of larger MT-immunopositive (mutant) patches than observed at 10 weeks, in our previous study.

Hence, prolonged $\lambda \mathrm{CgN}$ exposure may have cumulative effects upon mutant patch size. These effects could be related to $\lambda \mathrm{CgN}$ induced tissue injury in mouse colon (Donnelly et al, 2004), fission of immunopositive crypts and formation or enlargement of immunopositive patches, during continual or repeated regenerative healing.

While biomarkers of rate-limiting steps of tumorigenesis are informative, validation against tumour-associated end points is important. Aberrant crypt foci comprise a contiguous collection of crypts that have thickened epithelia, altered luminal openings and are clearly circumscribed from adjacent normal crypts (Bird, 1987). Gene mutations that are commonly observed in colon cancers including K-ras and APC are also observed in a proportion of ACF (Pretlow et al, 1993; Smith et al, 1994). Aberrant crypt foci are thus considered to represent earlystage colorectal tumorigenesis (Bird, 1987; Tudek et al, 1989; Takayama et al, 1998; Bird and Good, 2000), although large or persistent ACF may have greater cancer risk (Papanikolaou et al, 2000). The present study has shown that MTCRII may reflect combined effects of chemicals within a mixture, are induced in sufficient numbers to provide statistical power from relatively small animal samples and correlate with ACF formation at 20 weeks after the initiation of treatment. MTCRII may thus provide the basis for an intermediate risk assessment model for diet- or lifestyle-related genotoxic/nongenotoxic chemical combinations, relevant to colonic health.

\section{ACKNOWLEDGEMENTS}

This study was funded by Research Contract T01018 from the Food Standards Agency, which is gratefully acknowledged. 


\section{REFERENCES}

Bird RP (1987) Observation and quantification of aberrant crypts in the murine colon treated with a colon carcinogen: preliminary findings. Cancer Lett 37: 147-151

Bird RP, Good CK (2000) The significance of aberrant crypt foci in understanding the pathogenesis of colon cancer. Toxicol Lett 112-113: $395-402$

Burkart W, Jung T (1998) Health risks from combined exposures: mechanistic considerations on deviations from additivity. Mutat Res 411: $119-128$

Cook HA, Williams D, Thomas GA (2000) Crypt-restricted metallothionein immunopositivity in murine colon: validation of a model for studies of somatic stem cell mutation. J Pathol 191: 306-312

Donnelly ET, Bardwell H, Thomas GA, Williams ED, Hoper M, Crowe P, McCluggage WG, Stevenson M, Phillips DH, Hewer A, Osborne MR, Campbell FC (2004) Modulation of $N$-methyl- $N$-nitrosourea-induced crypt restricted metallothionein immunopositivity in mouse colon by a non-genotoxic diet-related chemical. Carcinogenesis 25: 847-855

Griffiths DF, Davies SJ, Williams D, Williams GT, Williams ED (1988) Demonstration of somatic mutation and colonic crypt clonality by $\mathrm{X}$ linked enzyme histochemistry. Nature 333: $461-463$

Herrero-Jimenez P, Thilly G, Southam PJ, Tomita-Mitchell A, Morgenthaler S, Furth EE, Thilly WG (1998) Mutation, cell kinetics, and subpopulations at risk for colon cancer in the United States. Mutat Res 400: 553 578

Jasani B, Campbell F, Navabi H, Schmid KW, Williams GT (1998) Clonal overexpression of metallothionein is induced by somatic mutation in morphologically normal colonic mucosa. J Pathol 184: 144-147

Kuraguchi M, Thomas GA, Williams ED (1997) Somatic mutation of the glucose-6-phosphate dehydrogenase (g6pd) gene in colonic stem cells and crypt restricted loss of G6PD activity. Mutat Res 379: 69-75
McLellan EA, Medline A, Bird RP (1991) Sequential analyses of the growth and morphological characteristics of aberrant crypt foci: putative preneoplastic lesions. Cancer Res 51: 5270-5274

Minamoto T, Mai M, Ronai Z (1999) Environmental factors as regulators and effectors of multistep carcinogenesis. Carcinogenesis 20: 519-527

OECD (2002) Guidance Notes for Analysis and Evaluation of Chronic Toxicity and Carcinogenicity Studies. Paris: Organisation for Economic Cooperation and Development, Environmental Directorate

Papanikolaou A, Wang QS, Papanikolaou D, Whiteley HE, Rosenberg DW (2000) Sequential and morphological analyses of aberrant crypt foci formation in mice of differing susceptibility to azoxymethane-induced colon carcinogenesis. Carcinogenesis 21: $1567-1572$

Park HS, Goodlad RA, Wright NA (1995) Crypt fission in the small intestine and colon. A mechanism for the emergence of G6PD locus-mutated crypts after treatment with mutagens. Am J Pathol 147: 1416-1427

Pretlow TP, Brasitus TA, Fulton NC, Cheyer C, Kaplan EL (1993) K-ras mutations in putative preneoplastic lesions in human colon. J Natl Cancer Inst 85: 2004-2007

Shpitz B, Hay K, Medline A, Bruce WR, Bull SB, Gallinger S, Stern H (1996) Natural history of aberrant crypt foci. A surgical approach. Dis Colon Rectum 39: $763-767$

Smith AJ, Stern HS, Penner M, Hay K, Mitri A, Bapat BV, Gallinger S (1994) Somatic APC and K-ras codon 12 mutations in aberrant crypt foci from human colons. Cancer Res 54: 5527-5530

Takayama T, Katsuki S, Takahashi Y, Ohi M, Nojiri S, Sakamaki S, Kato J, Kogawa K, Miyake H, Niitsu Y (1998) Aberrant crypt foci of the colon as precursors of adenoma and cancer. N Engl J Med 339: 1277-1284

Tudek B, Bird RP, Bruce WR (1989) Foci of aberrant crypts in the colons of mice and rats exposed to carcinogens associated with foods. Cancer Res 49: $1236-1240$ 